\title{
Eletrocardiograma na Síndrome de Haïssaguerre (Repolarização Precoce)
}

\author{
Electrocardiogram in Haïssaguerre Syndrome (Early Repolarization)
}

\author{
Raphael Chiarini1,2, Carlos Eduardo Duarte ${ }^{1,2, *}$, João Durval Ramalho Trigueiro Mendes Junior ${ }^{1,2}$, \\ J. Tarcísio Medeiros de Vasconcelos ${ }^{1,2}$, Silas dos Santos Galvão Filho ${ }^{1,2}$
}

ORCID IDs

Chiarini R (D) https://orcid.org/0000-0001-8619-8393

Duarte CE (D) https://orcid.org/0000-0001-6671-0820

Mendes Junior JDRT (D) https://orcid.org/0000-0002-9484-0013

Vasconcelos JTM (D) https://orcid.org/0000-0002-5152-2648

Galvão Filho SS (D) https://orcid.org/0000-0001-5236-164X

\section{RESUMO}

Introdução: O padrão de repolarização precoce (RP) tem sido tradicionalmente relacionado como uma variante benigna do eletrocardiograma (ECG). No entanto, desde 2008, quando dois estudos foram publicados por Haïssaguerre et al. e Rosso et al., com evidências de maior prevalência de RP em pessoas acometidas por fibrilação ventricular (FV) primária ou idiopática, esse paradigma tem sido contestado. Objetivo: Realizar uma profunda revisão acerca da RP e atual estado da arte acerca da estratificação de risco nesses pacientes. Métodos: Revisão da literatura acerca do tema avaliando os trabalhos publicados em revistas de alto impacto e a experiência dos especialistas sobre o assunto. Conclusão: A correlação de fatores de risco e o real valor dos vários métodos atualmente disponíveis como possíveis estratificadores de risco ainda são controversos. Avanços nas áreas da genética e biologia molecular podem futuramente auxiliar no entendimento da fisiopatologia e melhor estratificação de risco nessa população. Neste contexto, a padronização da definição e classificação da repolarização precoce mostra-se imperativa, uma vez que servirá de substrato para futuros estudos e pesquisas na área.

PALAVRAS-CHAVE: Fibrilação ventricular; Síndrome de Haïssaguerre; Eletrocardiografia; Risco de morte súbita; Repolarização precoce.

\begin{abstract}
Introduction: Early repolarization pattern (ERP) has traditionally been related as a benign variant of electrocardiography (ECG). However, since 2008, when two studies were published for Haïssaguerre et al. and Rosso et al., with evidence of a higher prevalence of ERP in people with primary or idiopathic ventricular fibrillation (VF), this paradigm has been challenged. Objective: To conduct a thorough review of early repolarization and current state of the art regarding risk stratification in these patients. Methods: Literature review on the subject evaluating the works published in high impact journals. Conclusion: The correlation of risk factors and the real value of the various methods currently available as possible risk stratifiers is still controversial. Advances in genetics and molecular biology may in the future help in understanding the pathophysiology and better risk stratification in this population. In this context, the standardization of the definition and classification of early repolarization is imperative, as it will serve as a substrate for future studies and researches in the area.
\end{abstract}

KEYWORDS: Ventricular fibrillation; Haïssaguerre syndrome, Electrocardiography; Sudden death risk; Early repolarization.

1.Beneficência Portuguesa de São Paulo, Eletrofisiologia e Estimulação Cardíaca artificial - São Paulo (SP) - Brasil.

2.Centro Avançado de Ritmologia e Eletrofisiologia, Eletrofisiologia e Estimulação Cardíaca Artificial - São Paulo (SP) - Brasil.

*Autor correspondente: carlosduarte@ritmologiacare.com.br

Recebido: 08 Nov 2019 | Aceito: 19 Nov 2019

Editor Associado: J. Tarcísio Medeiros de Vasconcelos 


\section{HISTÓRICO}

A presença de um padrão de repolarização precoce $(\mathrm{RP})$ em forma de entalhe ou empastamento da parte terminal do complexo QRS no eletrocardiograma (ECG) foi descrita pela primeira vez em 1936 por Shipley e Hallaran ${ }^{1}$. Porém a deflexão do ponto J que ocorre na junção do QRS com o segmento ST (também conhecida como onda de Osborn ou ondaJ) foi descrita pela primeira vez por Tomaszewski em $1938^{2}$. Por um longo período, houve um conceito consolidado de que a presença da RP no ECG não estava associada a qualquer adversidade. Esse conceito foi reconsolidado por Klatsky et al. ${ }^{3}$ em 2003, em um estudo envolvendo 73.088 pacientes submetidos a exame de saúde voluntário,incluindo ECG,em Oakland, Califórnia, entre 1983 e 1985. Os autores concluíram que a prevalência de RP em sua coorte foi de $0,9 \%$ (670/73.088), que os pacientes com $\mathrm{RP}$ eram menos propensos a experimentar arritmias e que a taxa geral de hospitalização e as consultas ambulatoriais não foram maiores do que na população controle.

Entretanto, na década de 2000, relatos clínicos, principalmente do Japão, demonstraram a presença de RP como único achado em pacientes sobreviventes de morte súbita cardíaca por fibrilação ventricular (FV) idiopática, sugerindo uma potencial natureza não benigna da RP. Evidências clínicas mais definitivas sobre essas observações vieram em 2007 e 2008 com Haïssaguerre et al. ${ }^{4}$ e Rosso et al..$^{5}$ respectivamente, em que a alta prevalência de $\mathrm{RP}$ foi associada a pacientes com $\mathrm{FV}$ idiopática. Haïssaguerre et al. ${ }^{4}$ evidenciaram a presença de RP em 31\% (64/206) dos casos de FV idiopática contra 5\% no grupo controle $(P<0,001)$. Rosso et al. ${ }^{5}$, em 2008, baseados em dados armazenados em cardiodesfibrilador implantável, observaram que 64 sobreviventes de FV idiopática com RP experimentaram maior recorrências de FV do que 142 sobreviventes de FV sem a presença de $\mathrm{RP}(41 \%$ vs $23 \%, P=0,008)$. Em 2015 , Macfarlane et al. ${ }^{6}$ publicaram um consenso para padronização do diagnóstico da RP. Em 2016, em um levantamento da American Heart Association, estimou-se que a incidência de $\mathrm{RP}$ na população geral poderia ser maior que $10 \%$. Frente às evidencias trazidas por publicações recentes, a American College of Cardiology (ACC), American Heart Association (AHA) e The Heart Rhythm Society (HRS) publicaram em conjunto um guia para manejo de pacientes com arritmias ventriculares e prevenção de morte súbita cardíaca incluindo os portadores da síndrome da repolarização precoce, trazendo novamente à tona a importância do tema.

\section{DEFINIÇÃO}

A definição de repolarização precoce $(\mathrm{RP})$ tem variado desde de sua primeira descrição por Tomaszewski em $1938^{2}$. Um consenso foi publicado recentemente em 2015 por Macfarlane et al. ${ }^{6}$ visando uniformizar os critérios para a correta definição e caracterização desse padrão. Ficou então definido que a RP pode se apresentar na forma de dois padrões distintos, sendo eles o padrão de entalhe e o padrão de empastamento. A RP está presente quando todos os seguintes critérios são encontrados:

1. Presença de entalhe ou empastamento (retardo) no ramo descente da onda $\mathrm{R}$. Se houver a presença de entalhe, este deve se localizar acima da linha de base, bem como o início do empastamento no final da onda $\mathrm{R}$.

2. O pico do entalhe no final do QRS (ponto Jp) ou o início do empastamento (ponto Jp) no final do QRS deve ser $\geq 0,1 \mathrm{mV}$ em duas ou mais derivações relacionadas no eletrocardiograma de 12 derivações, exceto de V1 a V3.

3. A duração do QRS deve ser < 120 ms.

Portanto, conforme esse consenso, a elevação isolada do segmento ST, na ausência de entalhe ou empastamento no ramo descendente da onda $\mathrm{R}$, não deve ser descrita como repolarização precoce.

Como forma de padronização visando estudos futuros e para a aplicação dos critérios de definição, recomenda-se a correta mensuração dos seguintes intervalos baseado em cada padrão de apresentação da RP (Fig. 1 $)^{6}$ :

- Padrão de entalhe:

1. A amplitude do ponto Ji no início do entalhe;

2. A amplitude do ponto Jp no pico do entalhe;

3. A amplitude do ponto Jt no término do entalhe;

4. A duração D1 do ponto Ji ao ponto Jp;

5. A duração D2 do ponto Ji ao ponto Jt.

- Padrão de empastamento:

1. A amplitude do ponto Jp no início do empastamento;

2. A amplitude do ponto Jt no término do empastamento;

3. A duração D2 do ponto Jp ao ponto Jt. 

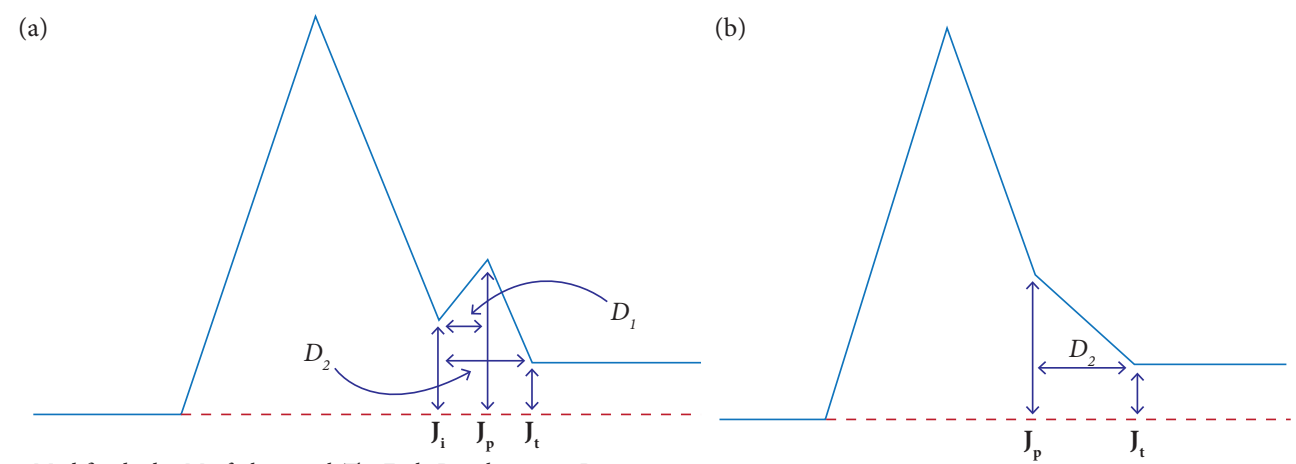

Modificado de : Macfarlane et al. The Early Repolarization Pattern: a consensus paper. 2015; 66:470-7

(a): Amplitude J inicial $\left(J_{i}\right)$, J pico $\left(J_{p}\right)$ e J termino $\left(J_{t}\right)$ e as durações $D_{1}$ e $D_{2}$ no padrão de entalhe.

(b): $\mathrm{J}_{\mathrm{p}}$ e $\mathrm{J}_{\mathrm{t}}$ e a duração $\mathrm{D}_{2}$ no padrão de empestamento, conforme definidos nos texto.

Figura 1. Padrões de entalhe e empastamento.

Outras recomendações em relação à mensuração:

\section{- Duração do QRS:}

É recomendado que a duração do QRS seja medida nas derivações que não apresentem o entalhe ou o empastamento, para que a duração do QRS não seja superestimada.

- Existência de empastamento final nos complexos QRS:

A confirmação da existência do empastamento ao final do QRS pode ser feita através da presença de um ângulo maior que 10 graus entre uma linha traçada ao final do QRS e no início do empastamento (Fig. ${ }^{6}{ }^{6}$ :

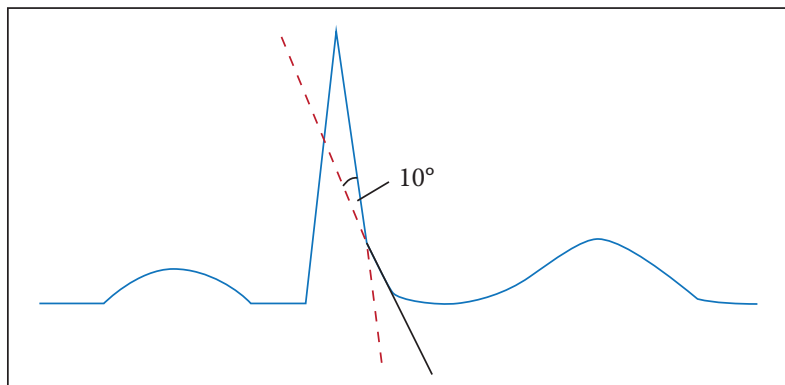

Modificado de : Macfarlane et al. The Early Repolarization Pattern: a consensus paper. $2015 ; 66: 470-7$

O empastamento pode ser determinado traçando uma linha ao final do QRS e outra no empastamento de o ângulo entre elas for maior que $10^{\circ}-=$ Confirmação de empastamento

Figura 2. Existência de empastamento.

\section{ETIOPATOGENIA}

Desde sua descrição, foram propostas muitas teorias acerca da compreensão do mecanismo precursor do fenótipo de $\mathrm{RP}$ e como ele pode predispor alguns pacientes portadores dessa alteração a um risco aumentado de arritmias. O entendimento da etiopatogenia requer a identificação e a caracterização detalhada do substrato eletrofisiológico no coração estruturalmente intacto dos pacientes portadores da síndrome de repolarização precoce.

O fenótipo clínico da síndrome RP é definido com base nas alterações na morfologia do ECG convencional de superfície corporal, conforme critérios já descritos neste estudo. Essas alterações no componente final do QRS com características tardias podem refletir tanto uma ativação tardia ou repolarização precoce; estas duas possibilidades não podem ser diferenciadas frente ao ECG de superfície convencional.

O nome síndrome de repolarização precoce foi dado com base em uma hipótese, não em um mecanismo subjacente confirmado. Portanto a primeira pergunta a ser respondida para o entendimento desse fenômeno é: as anormalidades no ECG em pacientes com RP são devidas ao atraso na ativação em regiões dos ventrículos ou devido à repolarização ventricular regional precoce? Buscando resposta para essa pergunta, Zhang et al. ${ }^{7}$ publicaram em 2017 um trabalho no qual pacientes portadores de RP foram submetidos a imagem eletrocardiográfica não invasiva (ECGI). Os potenciais do ECG foram adquiridos simultaneamente por 256 eletrodos usando uma aquisição de dados multicanal e, em seguida, os pacientes foram submetidos à tomografia computadorizada torácica com ECG para obtenção da geometria epicárdica e posições dos eletrodos no tórax (Fig. 3) 3 $^{8}$.

O ECGI não invasivo revelou a presença de um substrato eletrofisiológico anormal em pacientes com RP, caracterizado por encurtamento da duração do potencial de ação, gradientes acentuados de repolarização e distribuição regional dos eletrogramas da onda J no epicárdico. Gradientes 
de repolarização acentuados fornecem substrato suscetível ao desenvolvimento de condução assimétrica e arritmias reentrantes. Foi possível a correlação de locais de origem de extrassístoles ventriculares em regiões com presença de onda J e gradientes acentuados de repolarização mapeado durante ritmo sinusal (Fig. 4$)^{7}$.

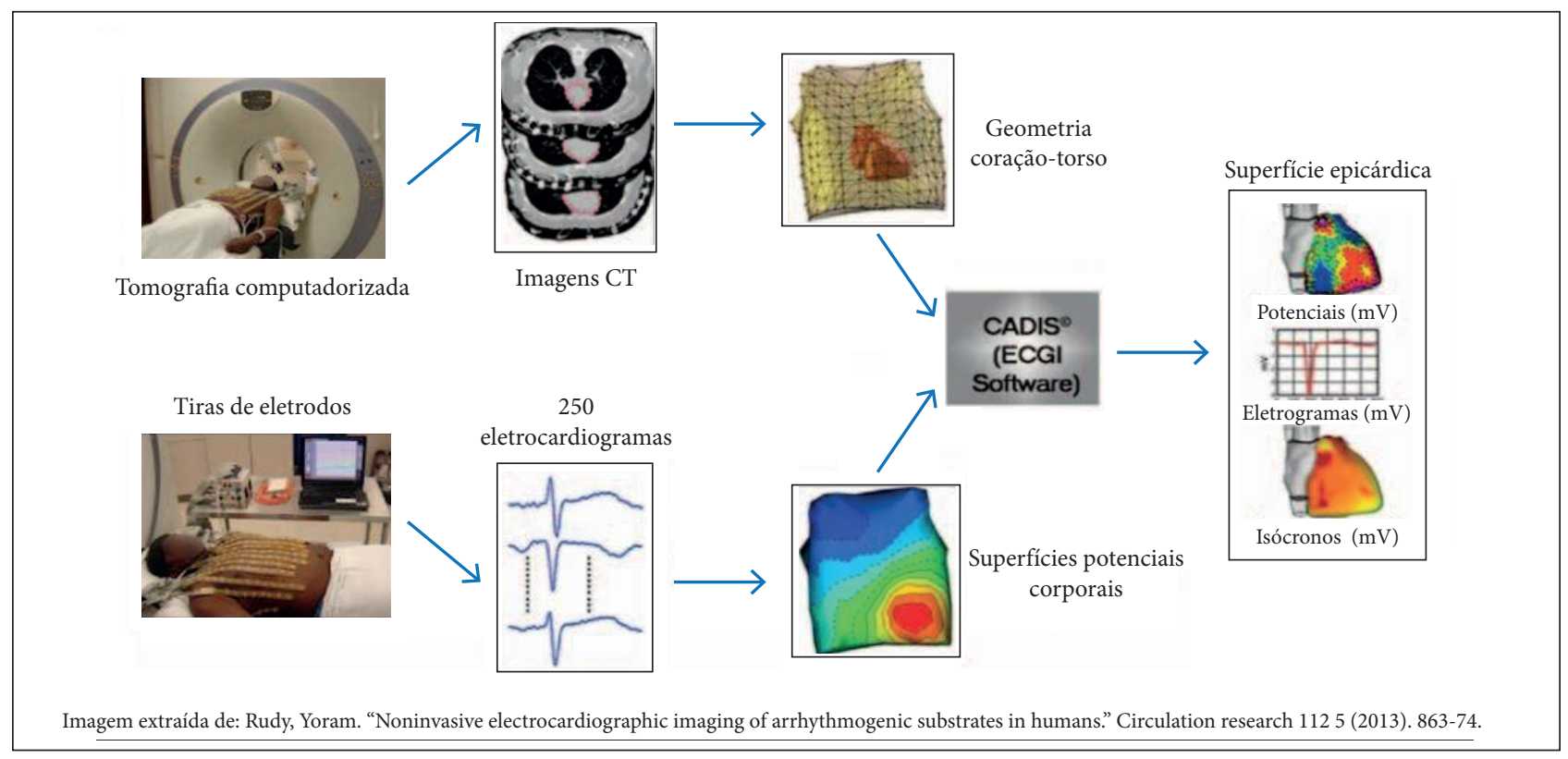

Figura 3. ECG com 250 eletrodos e construção da imagem epicárdica com auxílio da tomografia

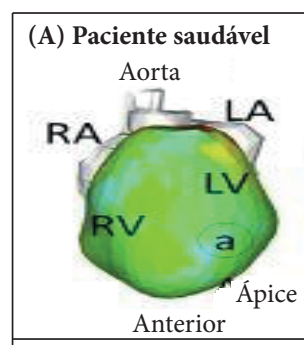

(C) Paciente Sala-14

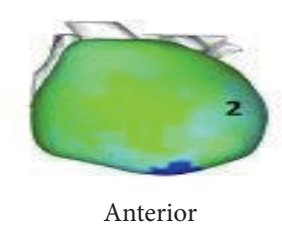

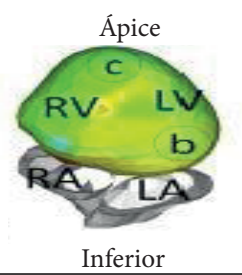

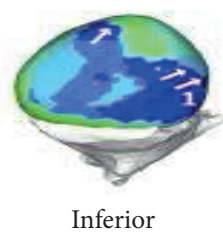

(B) Paciente Sala-11

(E) Eletrogramas
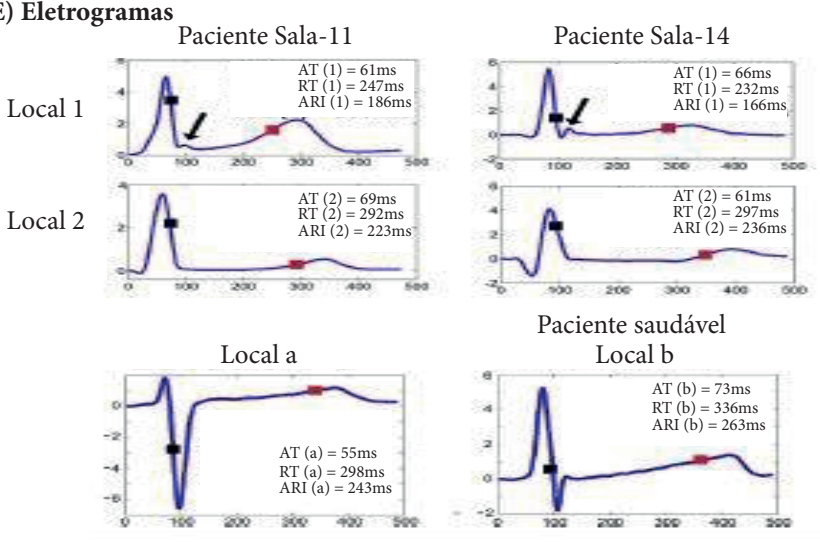

Paciente saudável

Local b

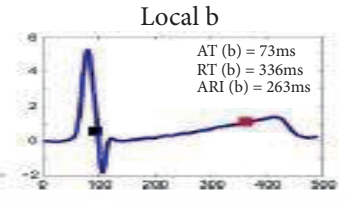

(D) Paciente Sala-20

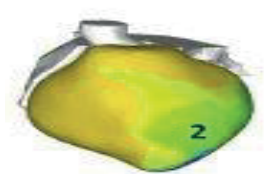

Anterior

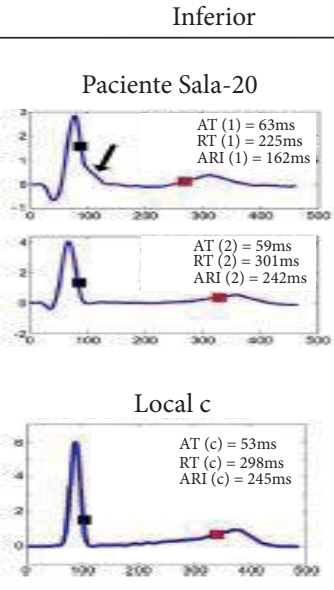

Modificado de:

Zhang et al. The substrate

of Early Repolarization

Syndrome. JACC: Clinical Electrophysiology. Mar 2017. 


\section{CLASSIFICAÇÃO CLÍNICA E ELETROCARDIOGRÁFICA}

Podemos classificar a manifestação da repolarização precoce em relação à sua manifestação clínica ou apresentação eletrocardiográfica.

Clinicamente, podemos dividir em duas apresentações:

- Sindrome da repolarização precoce (Sindrome de Haïssaguerre): elevação do ponto $\mathrm{J} \geq 0,1 \mathrm{mV}$ em duas derivações relacionadas, inferior ou lateral, com um entalhe ou empastamento do QRS, em pacientes com relato de morte súbita recuperada, $\mathrm{FV}$ documentada ou taquicardia ventricular polimórfica, ou com padrão familiar (mutação genética).

- Padrão de repolarização precoce: presença da alteração eletrocardiográfica: elevação do ponto $\mathrm{J} \geq 0,1 \mathrm{mV}$ em duas derivações relacionadas, inferior ou lateral, com um entalhe (onda J) ou empastamento no final do QRS.

Quanto à apresentação eletrocardiográfica além dos padrões já descritos de entalhe e empastamento, conforme pode ser visto nas Figs. 5 e 6, deve-se classificar a RP em relação ao segmento ST em:

- Repolarização precoce com segmento ST ascendente;

- Repolarização precoce com segmento ST horizontal;

- Repolarização precoce com segmento ST descendente ${ }^{6}$.

\section{IMPORTÂNCIA CLÍNICA}

O padrão de repolarização precoce tem sido tradicionalmente relacionado como uma variante benigna do ECG e chegou a ser considerado como um marcador de

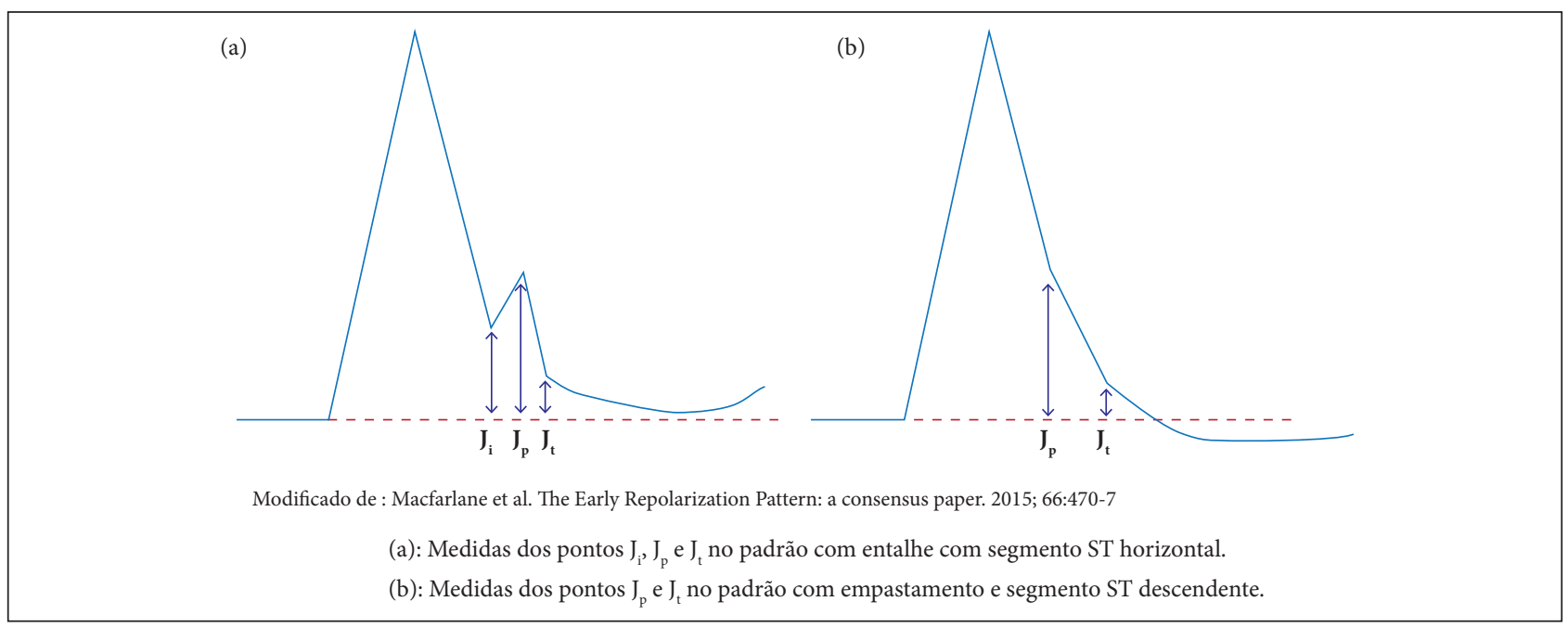

Figura 5. Medidas do entalhe em padrão descendente e do empastamento em padrão descendente do ST.

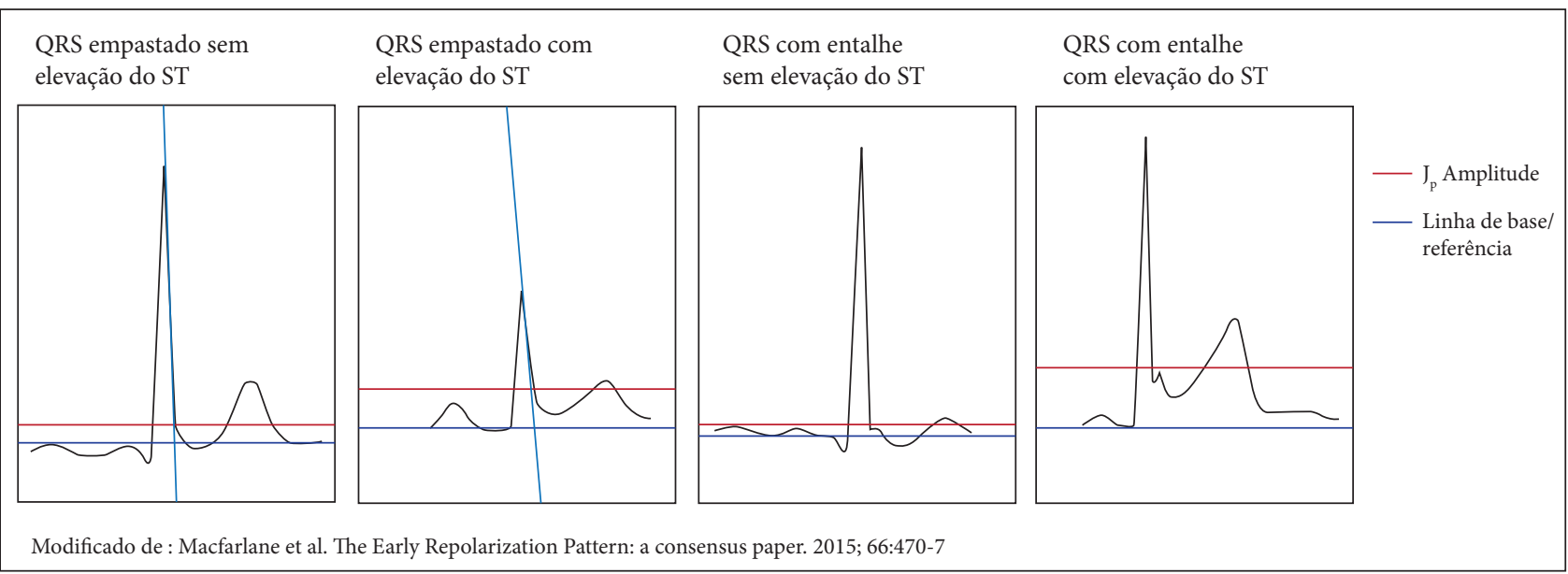

Figura 6. Avaliação do ponto Jp em QRS com e sem elevação do segmento ST. 


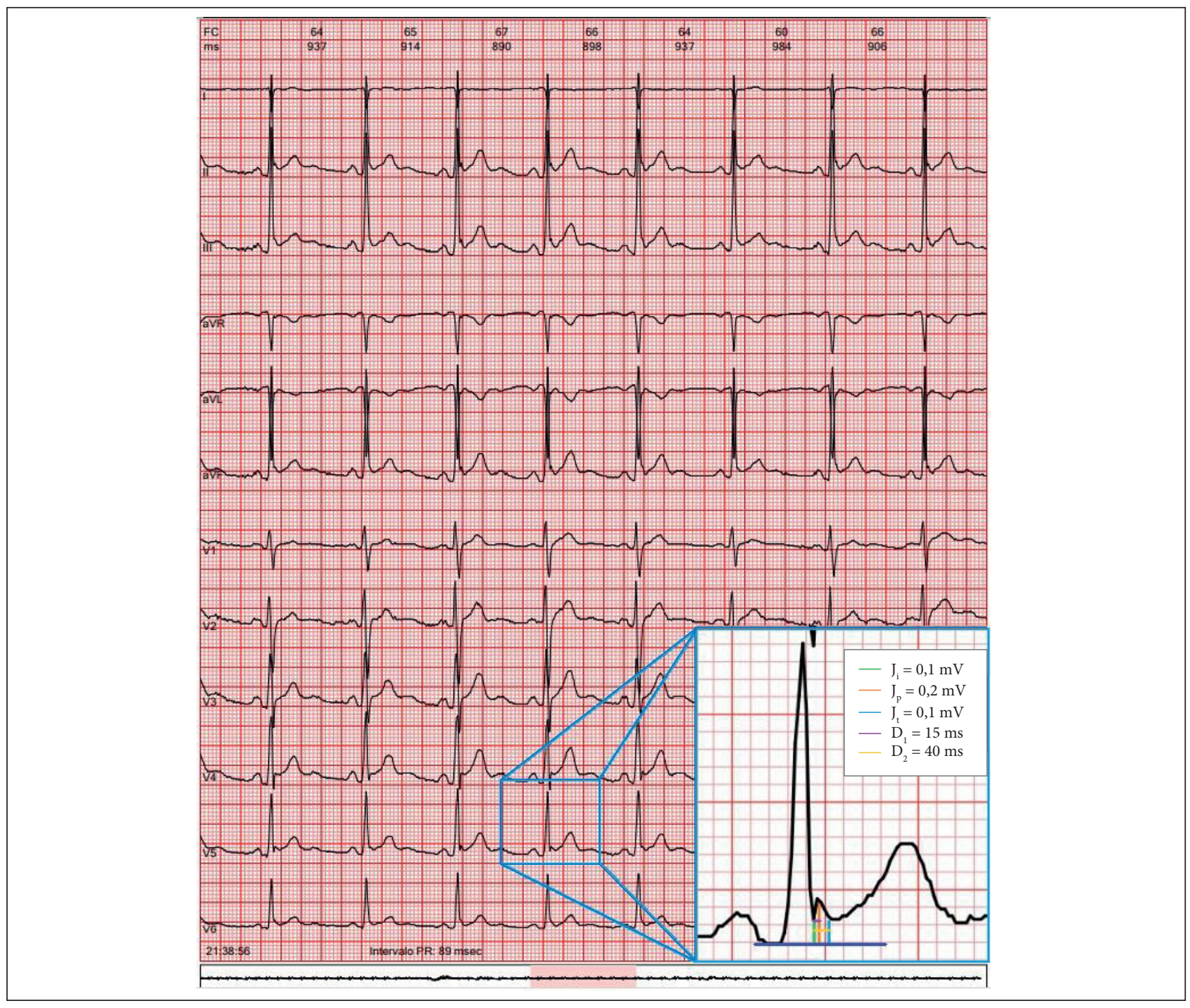

Figura 7. Exemplos ECG e VCG.

boa saúde, por estar presente em jovens e atletas. Estima-se que a prevalência do padrão de RP na população geral esteja entre 1 e 13\%, com maior prevalência relatada entre pacientes jovens. No entanto, desde 2008, quando dois estudos foram publicados por Haïssaguerre et al. ${ }^{9}$ e Rosso et al. ${ }^{5}$, com evidências de maior prevalência de RP em pessoas acometidas por fibrilação ventricular $(\mathrm{FV})$ primária ou idiopática, esse paradigma tem sido contestado. Rosso et al. ${ }^{5}$ demonstraram maior prevalência da elevação do ponto J no grupo de pacientes sobreviventes de morte súbita em comparação com o grupo de atletas jovens e adultos do grupo controle $^{5}$ (Fig. 8), e também correlacionou as derivações nas quais a evidência de RP apresentou maior relação com episódios de FV idiopática (Fig. 9).

Em 2015, Mahida et al. ${ }^{10}$ publicaram um estudo em que foram selecionados 81 pacientes com padrão de RP ao ECG que experimentaram episódio de morte súbita abortada por $\mathrm{FV}$, sendo então todos portadores de cardiodesfibrilador implantável. Esses pacientes foram selecionados de um registro internacional de $\mathrm{FV}$ idiopática contendo 367 pacientes. Tais pacientes foram submetidos a protocolos padrão de estudo eletrofisiológico (EEF) para indução de FV e em seguida por interrogação seriada do cardiodesfibrilador implantável. Nesse grupo, foi possível a indução de FV durante o EEF em apenas 18 dos 81 (22\%) pacientes; 6 dos 18 (33\%) pacientes apresentaram novos episódios de FV registrados pelo cardiodesfibrilador implantável durante o seguimento. Em relação ao grupo de pacientes em que não foi possível a indução de FV durante o EEF, 21 dos 63 (33\%) experimentaram novos episódios de FV documentados pelo cardiodesfibrilador implantável ao longo do seguimento. Demonstrando que os atuais protocolos de estimulação durante o estudo eletrofisiológico não aumentaram a estratificação de risco de fibrilação ventricular 


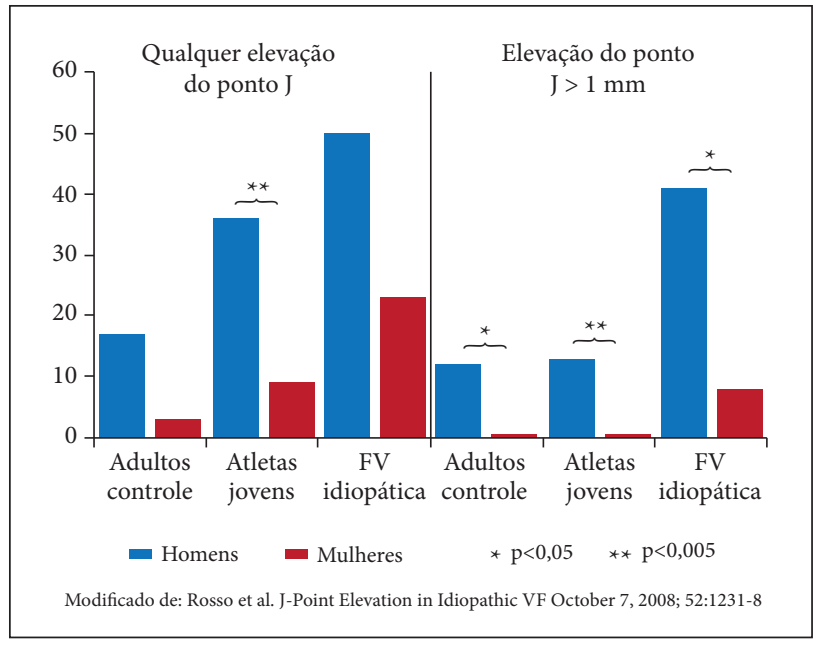

Figura 8. Influência do gênero da incidência de elevação do ponto J em diferentes grupos de pacientes

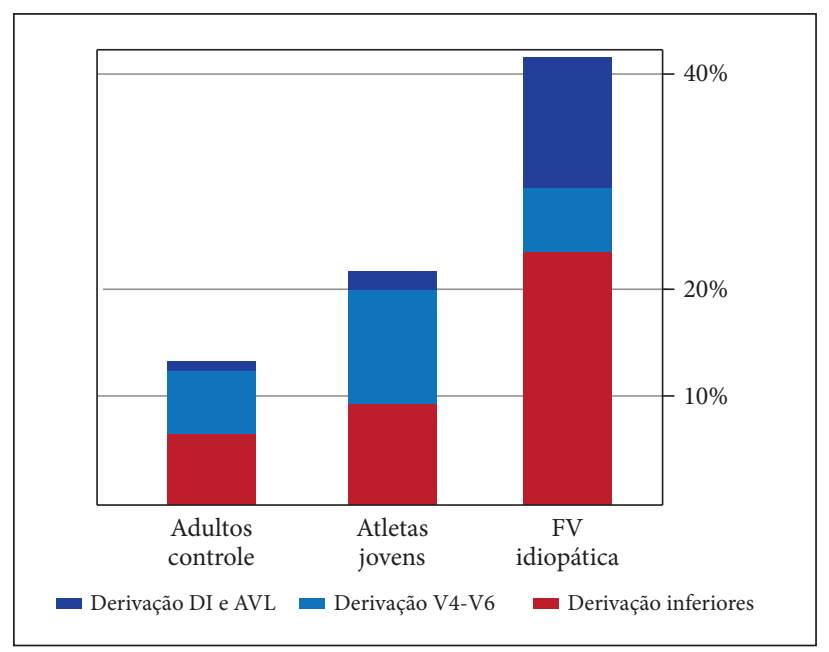

Figura 9. Distribuição da prevalência da onda J nas derivações nos diferentes grupos de pacientes.

nos portadores da Síndrome de Haïssaguerre.Em contrapartida, em análise retrospectiva, Mahida et al. ${ }^{10}$ propuseram fatores de risco correlacionados nessa população estudada como possíveis preditores de risco para morte súbita (Fig. 10) ${ }^{10}$ :

Em 2017, baseados em revisão de publicações recentes, a American College of Cardiology (ACC),American Heart Association (AHA) e The Heart Rhythm Society (HRS) publicaram em conjunto um guia para manejo de pacientes com arritmias ventriculares e prevenção de morte súbita cardíaca ${ }^{11}$ incluindo portadores da síndrome da repolarização precoce (Tabela 1).

\section{CONCLUSÃO}

Embora vários estudos tenham demonstrado a relação entre a presença de repolarização precoce e um aumento

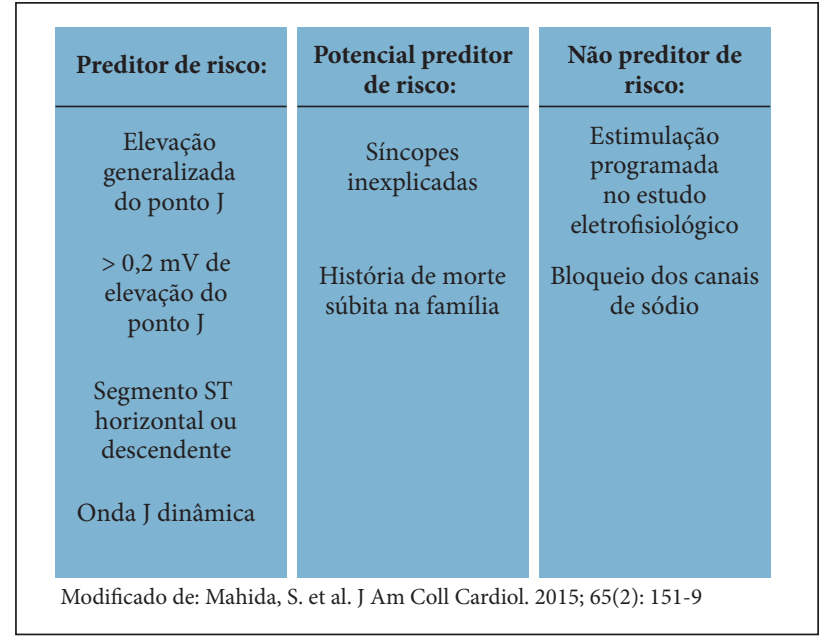

Figura 10. Achados no presente estudo no contexto de fatores de risco previamente identificados para morte súbita em portadores da síndrome da repolarização precoce.

Tabela 1. Recomendações para a Síndrome da Repolarização Precoce.

\begin{tabular}{ccc}
\hline CR & NE & Recomendação \\
\hline I & B-NR & $\begin{array}{c}\text { 1. Em pacientes assintomáticos com padrão } \\
\text { de RP ao ECG, observação sem nenhum } \\
\text { tratamento é recomendada. }\end{array}$ \\
\hline I B-NR & $\begin{array}{c}\text { 2. Em pacientes com padrão de RP ao ECG } \\
\text { sustentada, é recomendado o implante de } \\
\text { cardiodesfibrilador. }\end{array}$ \\
\hline B-NR & $\begin{array}{l}\text { 3. Em pacientes com padrão de RP ao ECG, } \\
\text { não são recomendados testes genéticos. }\end{array}$ \\
\hline
\end{tabular}

CR: Classe de Recomendação; NE: Nível de evidência'².

da incidência de morte súbita cardíaca, a correlação de fatores de risco e o real valor dos vários métodos atualmente disponíveis como possíveis estratificadores de risco ainda são controversos. Avanços nas áreas da genética e da biologia molecular podem futuramente auxiliar no entendimento da fisiopatologia e melhor estratificação de risco nesta população. Nesse contexto, a padronização da definição e classificação da repolarização precoce mostra-se imperativa, uma vez que servirá de substrato para futuros estudos e pesquisas na área.

\section{CONTRIBUIÇÃO DOS AUTORES}

Todos os autores contribuiram igualmente para este artigo. 


\section{REFERÊNCIAS}

1. Shipley RA, Hallaran WR. The four lead electrocardiogram in 200 normal men and women. Am Heart J. 1936;11(3):32545. https://doi.org/10.1016/S0002-8703(36)90417-9

2. Tomaszewski W. Changement electrocardiographiques observes chez un homme mort de froid. Arch Mal Coeur Vaiss. 1938;31:525-528.

3. Klatsky AL, Oehm R, Cooper RA, Udaltsova N, Armstrong MA. The early repolarization normal variant electrocardiogram: correlates and consequences. Am J Med. 2003;115(3):171-7. https://doi.org/10.1016/s0002-9343(03)00355-3

4. Haïssaguerre M, Sacher F, Derval N, Jesel L, Deisenhofer I, Roy L, et al. Early repolarization in the inferolateral leads: A new syndrome associated with sudden cardiac death. I Interv Card Electrophysiol. 2007;18(3):281. https://doi.org/10.1007/s10840-007-9136-7

5. Rosso R, Kogan E, Belhassen B, Rozovski U, Scheinman MM, Zeltser D, et al. J-point elevation in survivors of primary ventricular fibrillation and matched control subjects: incidence and clinical significance. J Am Coll Cardiol. 2008;52(15):1231 8. https://doi.org/10.1016/j.jacc.2008.07.010

6. Macfarlane PW, Antzelevitch C, Haïssaguerre M, Huikuri HV, Potse M, Rosso R, et al. The early repolarization pattern: a consensus paper. J Am Coll Cardiol. 2015;66(4):470-7. https://doi.org/10.1016/j. jacc.2015.05.033

7. Zhang J, Hocini M, Strom M, Cuculich PS, Cooper DH, Sacher F, et al. The Electrophysiological Substrate of Early Repolarization Syndrome - Noninvasive Mapping in Patients. JACC: Clinical Electrophysiology. 2017;3(8):894-904. https://doi.org/10.1016/j. jacep.2016.12.017

8. Yoram R. Noninvasive electrocardiographic imagin of arrhythmogenic substrates in humans. Circ Res. 2013;125(5):96374. https://doi.org/10.1161/CIRCRESAHA.112.279315

9. Haïssaguerre M, Derval N, Sacher F, Jesel L, Deisenhofer I, Roy L, et al. Sudden cardiac arrest associated with early repolarization. N Engl J Med. 2008;358(19):2016-23. https://doi.org/10.1056/ NEJMoa071968

10. Mahida S, Derval N, Sacher F, Leenhardt A, Deisenhofer I, Babuty $D$, et al. Role of electrophysiological studies in predicting risk of ventricular arrhythmia in early repolarization syndrome. J Am Coll Cardiol. 2015;65(2):151-9. https://doi.org/10.1016/j. jacc.2014.10.043

11. Haïssaguerre M, Sacher F, Nogami A, Komiya N, Bernard A, Probst $\checkmark$, et al. Characteristics of recurrent ventricular fibrillation associated with inferolateral early repolarization role of drug therapy. J Am Coll Cardiol. 2009;53(7):612-9. https://doi.org/10.1016/j. jacc.2008.10.044

12. Al-Khatib SM, Stevenson WG, Ackerman MJ, Bryant WJ, Callans DJ, Curtis AB, et al. 2017 AHAACC/HRS guideline for management of patients with ventricular arrhythmias and the prevention of sudden cardiac death, Heart Rhythm. 2017;15(10):e73-189. https://doi. org/10.1016/j.hrthm.2017.10.036
13. TikkanenJT,Anttonen O,Junttila MJ,Aro AL, KerolaT, Rissanen HA, et al. Long-term outcome associated with early repolarization on electrocardiography. N Engl J Med. 2009;361(26):2529-37. https://doi.org/10.1056/NEJMoa0907589

14. Mehta MC, Jain AC. Early repolarization on scalar electrocardiogram. Am J Med Sci. 1995;309(6):305-11. https://doi.org/10.1097/00000441-199506000-00001

15. Kambara H, Phillips J. Long-term evaluation of early repolarization syndrome (normal variant RS-T segment elevation). Am J Cardiol. 1976;38(2):157-6. https://doi.org/10.1016/0002-9149(76)90142-9

16. Nam GB, Kim YH, Antzelevitch C. Augmentation of J waves and electrical storms in patients with early repolarization. New EnglJ Med. 2008;358(19):2078-9. https://doi.org/10.1056\%2FNEJMc0708182

17. Cappato R, Furlanello F, Giovinazzo V, Infusino T, Lupo P, Pittalis M, et al. J wave, QRS slurring, and ST elevation in athletes with cardiac arrest in the absence of heart disease: marker of risk or innocent bystander? Circ Arrhythm Electrophysiol 2010;3(4):30511. https://doi.org/10.1161/CIRCEP.110.945824

18. Miyazaki S, Shah AJ, Haïssaguerre M. Early repolarization syndrome - a new electrical disorder associated with sudden cardiac death. Jpn Circ J. 2010;74(10):2039-44. https://doi.org/10.1253/circj.CJ-10-0753

19. Adhikarla C, Boga M, Wood AD, Froelicher VF. Natural history of the electrocardiographic pattern of early repolarization in ambulatory patients. Am J Cardiol. 2011;108(12):1831-5. https://doi.org/10.1016/j.amjcard.2011.07.055

20. Siebermair J, Sinner MF, Beckmann BM, Laubender RP, Martens E, Sattler S, et al. Early repolarization pattern is the strongest predictor of arrhythmia recurrence in patients with idiopathic ventricular fibrillation: results from a single centre long-term follow-up over 20 years. Europace. 2016;18:718-25. https://doi.org/10.1093/europace/euv301

21. Sinner MF, Porthan K, Noseworthy PA, Havulinna AS, Tikkanen $J T$, Müller-Nurasyid M, et al. A meta-analysis of genomewide association studies of the electrocardiographic early repolarization pattern. Heart Rhythm. 2012;9(10):1627-34. https://doi.org/10.1016/j.hrthm.2012.06.008

22. Tikkanen JT, Wichmann V, Junttila MJ, Rainio M, Hookana E, Lappi O-P, et al. Association of early repolarization and sudden cardiac death during an acute coronary event. Circ Arrhythm Electrophysiol. 2012;5(4):714-8. https://doi.org/10.1161/CIRCEP.112.970863

23. Junttila MJ, TikkanenJT, Kenttä T, Anttonen O, Aro AL, Porthan K, et al. Early repolarization as a predictor of arrhythmic and nonarrhythmic cardiac events in middle-aged subjects. Heart Rhythm. 2014;11(10):1701-6. https://doi.org/10.1016/j.hrthm.2014.05.024

24. Cheng YJ, Lin XX, Ji CC, Chen XM, Liu LJ, Tang K, et al. Role of early repolarization pattern in increasing risk of death. J Am Heart Assoc. 2016;5(9):1-10. https://doi.org/10.1161/JA 